\title{
Search for Varying Constants of Nature from Astronomical Observation of Molecules
}

\author{
Wim Ubachs ${ }^{1}$
}

Received: 20 April 2017 / Accepted: 10 October 2017 / Published online: 30 November 2017

(C) The Author(s) 2017. This article is published with open access at Springerlink.com

\begin{abstract}
The status of searches for possible variation in the constants of nature from astronomical observation of molecules is reviewed, focusing on the dimensionless constant representing the proton-electron mass ratio $\mu=m_{p} / m_{e}$. The optical detection of $\mathrm{H}_{2}$ and $\mathrm{CO}$ molecules with large ground-based telescopes (as the ESO-VLT and the Keck telescopes), as well as the detection of $\mathrm{H}_{2}$ with the Cosmic Origins Spectrograph aboard the Hubble Space Telescope is discussed in the context of varying constants, and in connection to different theoretical scenarios. Radio astronomy provides an alternative search strategy bearing the advantage that molecules as $\mathrm{NH}_{3}$ (ammonia) and $\mathrm{CH}_{3} \mathrm{OH}$ (methanol) can be used, which are much more sensitive to a varying $\mu$ than diatomic molecules. Current constraints are $|\Delta \mu / \mu|<5 \times 10^{-6}$ for redshift $z=2.0-4.2$, corresponding to look-back times of 10-12.5 Gyrs, and $|\Delta \mu / \mu|<1.5 \times 10^{-7}$ for $z=0.88$, corresponding to half the age of the Universe (both at $3 \sigma$ statistical significance). Existing bottlenecks and prospects for future improvement with novel instrumentation are discussed.
\end{abstract}

Keywords Varying constants $\cdot$ Extragalactic astronomy $\cdot$ Molecular spectroscopy

\section{Introduction}

Extensions of the Standard Model (SM) of physics can occur in various ways. One possibility of such an extension is the temporal or spatial variation of fundamental constants of nature, such as the coupling strengths of the known four forces, the strong and weak forces, electromagnetism and gravity. Their coupling strengths are inserted in the SM as

High Performance Clocks with Special Emphasis on Geodesy and Geophysics and Applications to Other Bodies of the Solar System

Edited by Rafael Rodrigo, Véronique Dehant, Leonid Gurvits, Michael Kramer, Ryan Park and Peter Wolf

\footnotetext{
$\triangle$ W. Ubachs

w.m.g.ubachs@vu.nl

1 Department of Physics and Astronomy, Vrije Universiteit Amsterdam, De Boelelaan 1081, 1081 HV Amsterdam, The Netherlands
} 
free parameters, as are the relative masses of the elementary particles. However, in order to describe physics in a consistent framework of quantum field theories it does not suffice to allow the fundamental constants to vary at will. For consistency, and to retain e.g. conservation of energy, the inclusion of additional fields is required, often referred to as 'dilaton' fields that may in the simplest form be of scalar nature (Barrow et al. 2002). Such fields may be regarded as additional forces or 'quintessence', and may be associated with other unknown forms of (dark) matter or energy. If such connection exists, then the values of some constants of nature might follow the increasing amount of dark energy in the Universe, in which case the variation of constants would manifest itself on a cosmological time scale. Alternatively, the fundamental constants might be connected to local effects such as matter density (Khoury and Weltman 2004) or gravitational fields (Flambaum and Shuryak 2008). Theories involving variation of fundamental constants exist in various flavors and are e.g. described in the review by Uzan (2011).

The quantum mechanical energy level structure of atoms and molecules is determined by the value of the fine structure constant $\alpha$ and the proton-to-electron mass ratio $\mu=m_{p} / m_{e}$ (Kozlov and Levshakov 2013; Jansen et al. 2014). Searches for variation of both dimensionless constants $\alpha$ and $\mu$ focus primarily on spectroscopic methods. Comparison between spectra obtained from astronomical observations at high redshift with the same spectra measured at high precision in the present epoch with laboratory-based methods may reveal minute shifts in the values of those constants. Spectroscopy, i.e. the measurement of wavelengths or frequencies of absorption lines, is a solid method in view of the accuracy at which such quantities can be measured. In the analysis procedures of comparing spectra the small differential shifts of absorption lines imposed by varying constants must be disentangled from the cosmological and gravitational redshifts involved in astronomical observations. However, General Relativity (GR) predicts that these latter redshifts are dispersionless, hence disentangling is straightforward. As variation of constants implies a breakdown of Einstein's equivalence principle, a corner stone of existing theories of physics, any resulting differential or dispersion effect may be considered as a token for new physics.

The search for a varying fine structure constant $\alpha$ from comparison of spectroscopic lines was pursued already in the 1950s (Savedoff 1956) based on the alkali-doublet method. Here it is used that the separation of two spectral lines probing a spin-orbit doublet from a common ground state scales with $\alpha^{2}$. Bahcall and Schmidt (1967) therewith constrained a relative variation of $\alpha$ at the $10^{-3}$ level from observations of a pair O III emission lines at redshift $z=0.2$. The accuracy was much improved by the Many-Multiplet-(MM) method, where absolute transition frequencies of various elements can be used simultaneously for probing a variation of $\alpha$. The MM-method requires detailed ab initio calculations of atomic structure to derive sensitivity coefficients (Dzuba et al. 1999). Such sensitivity coefficients reflect by how much spectral lines will shift as a result of a relative change in the fine structure constant $\Delta \alpha / \alpha$, and they appear to be largest for heavy elements where electrons undergo relativistic motion. Based on the MM-method and observations with large 8-10 m class telescopes certain indications of a temporally (Webb et al. 2001) and spatially (Webb et al. 2011) varying $\alpha$ were reported. It is discussed in the scientific literature whether these effects might be due, at least in part, to calibration issues with the spectrographs connected to the large optical telescopes used to probe varying $\alpha$ at high redshift (Rahmani et al. 2013; Whitmore and Murphy 2015; Dumont and Webb 2017).

Focus of this paper is on another dimensionless constant, the proton-to-electron mass ratio $\mu$, and the constraints that can be derived on its relative variation $\Delta \mu / \mu$. In theoretical models based on Grand Unification it was shown that the rate of change in $\mu$ is much faster than a putative rate of change in $\alpha$ (Calmet and Fritzsch 2002; Langacker et al. 2002), thus 
Table 1 List of the ten high redshift $\mathrm{H}_{2}$ absorption systems analyzed so far for $\mu$-variation. The identification of the quasar source, the redshift $z$ of the absorbing system, and the $\mathrm{J} 2000$-coordinates of the quasar source are listed. The column densities $N\left(\mathrm{H}_{2}\right)$ are given on a $\log _{10}$ scale in $\mathrm{cm}^{-2} . R_{\text {mag }}$ represents the magnitude in the visible range

\begin{tabular}{|c|c|c|c|c|c|c|}
\hline Quasar & $z_{\mathrm{abs}}$ & RA(J2000) & Decl.(J2000) & $N\left(\mathrm{H}_{2}\right)$ & $R_{\text {mag }}$ & Refs. \\
\hline HE0027-1836 & 2.42 & $00: 30: 23.62$ & $-18: 19: 56.0$ & 17.3 & 17.37 & Rahmani et al. (2013) \\
\hline Q0347-383 & 3.02 & $03: 49: 43.64$ & $-38: 10: 30.6$ & 14.5 & 17.48 & $\begin{array}{l}\text { Ivanchik et al. (2005), } \\
\text { Reinhold et al. (2006), } \\
\text { King et al. (2008), } \\
\text { Wendt and Molaro (2012), } \\
\text { Thompson et al. (2009) }\end{array}$ \\
\hline Q0405-443 & 2.59 & 04:07:18.08 & $-44: 10: 13.9$ & 18.2 & 17.34 & $\begin{array}{l}\text { Ivanchik et al. (2005), } \\
\text { Reinhold et al. (2006), } \\
\text { King et al. (2008), } \\
\text { Thompson et al. (2009) }\end{array}$ \\
\hline Q0528-250 & 2.81 & 05:30:07.95 & $-25: 03: 29.7$ & 18.2 & 17.37 & King et al. $(2008,2011)$ \\
\hline B0642-5038 & 2.66 & $06: 43: 26.99$ & $-50: 41: 12.7$ & 18.4 & 18.06 & $\begin{array}{l}\text { Bagdonaite et al. (2014b), } \\
\text { Albornoz Vásquez et al. (2014) }\end{array}$ \\
\hline Q1232+082 & 2.34 & $12: 34: 37.58$ & $+07: 58: 43.6$ & 19.7 & 18.40 & $\begin{array}{l}\text { Varshalovich et al. (2001), } \\
\text { Ivanchik et al. (2010), } \\
\text { Daprá et al. (2017b) }\end{array}$ \\
\hline $\mathrm{J} 1237+064$ & 2.69 & $12: 37: 14.60$ & $+06: 47: 59.5$ & 19.2 & 18.21 & Daprà et al. (2015) \\
\hline J1443+2724 & 4.22 & $14: 43: 31.18$ & $+27: 24: 36.4$ & 18.3 & 18.81 & Bagdonaite et al. (2015) \\
\hline J2123-0050 & 2.06 & $21: 23: 29.46$ & $-00: 50: 52.9$ & 17.6 & 15.83 & $\begin{array}{l}\text { Malec et al. (2010), } \\
\text { van Weerdenburg et al. (2011) }\end{array}$ \\
\hline Q2348-011 & 2.42 & $23: 50: 57.87$ & $-00: 52: 09.9$ & 18.4 & 18.31 & Bagdonaite et al. (2012) \\
\hline
\end{tabular}

making $\mu$ a sensitive test ground for probing varying constants. Typically the constant $\mu$ is associated with the quantized motion of nuclei in molecules, through their intramolecular rotation, vibration and tunneling modes. Again for any model comparing astronomical spectra with zero-redshift laboratory data, sensitivity coefficients $K_{i}=(d \ln \lambda) /(d \ln \mu)$ must be evaluated for each allowed quantum transition in a molecule (Jansen et al. 2014). In the following the results of ground-based observation of molecular hydrogen and carbon monoxide at high-redshift will be presented, yielding constraints on $\Delta \mu / \mu$. It will further be discussed how vacuum-ultraviolet (VUV) absorption by $\mathrm{H}_{2}$ in galactic white dwarf objects, detected by a satellite-based spectrograph, will be used for constraining a possible dependence of $\mu$ on a gravitational field. The microwave detection of molecular absorption used for probing a varying $\mu$ is described in a subsequent section. Future developments in optical and radio astronomy, as well as bottlenecks, challenges and opportunities for more tight constraints on variation of the proton-electron mass ratio will be discussed in a concluding section.

\section{Optical Detection of the $\mathrm{H}_{2}$ Molecule}

Hydrogen is by far the most abundant species in Galaxies and is found in both atomic and molecular forms in the interstellar medium. The investigation of $\mathrm{H}_{2}$ absorption in galaxies at high redshift in the line-of-sight towards quasars favorably draws from this omnipresence of $\mathrm{H}_{2}$. Nevertheless, although large numbers of quasars have been detected, many of which with a Damped-Lyman- $\alpha$ (DLA) absorption feature marking a column density for atomic 
hydrogen in excess of $2 \times 10^{20} \mathrm{~cm}^{-2}$, only a limited number of systems with pronounced $\mathrm{H}_{2}$ absorption have been identified. Currently some $23 \mathrm{H}_{2}$ absorbers at redshifts $z>2$ have been found by various authors and a compilation can be found in Ubachs et al. (2016). An additional set of 23 systems with tentative absorptions have been detected (Balashev et al. 2014). In those systems typically a column density for $\mathrm{H}_{2}$ is detected at the $1 \%$ level with respect to that of $\mathrm{H}$, although much higher molecular fractions have also been detected recently, see e.g. Noterdaeme et al. (2017).

The strongest electric dipole-allowed transitions in the $\mathrm{H}_{2}$ molecule, those of the Lyman and Werner band systems, occur at rest-frame wavelengths of 912-1150 , so that a significant fraction of the absorption lines shift into the atmospheric transmission win$\operatorname{dow}(\lambda>3050 \AA)$ for absorption redshifts of $z>2$. Since the molecular absorption bands lay shortward in wavelength of the Lyman- $\alpha$ line (1215 $\AA$ ), $\mathrm{H}_{2}$ line absorptions are typically found in the 'Lyman- $\alpha$ forest'. This consists of a spectral region crowded with many Lyman$\alpha$ absorption lines, produced by the intergalactic gas over the full range of redshifts along the line of sight, from the observer to the background quasar (Rauch 1998). This Lyman- $\alpha$ forest produces in fact a random spectrum overlaying the spectral region where $\mathrm{H}_{2}$ absorption lines are located, thus forming an obstacle to be overcome in the data analysis (Malec et al. 2010).

Currently some ten $\mathrm{H}_{2}$ absorptions systems, listed in Table 1, have been analyzed for $\mu$-variation. These are good quality systems in view of brightness of the background quasar, typically in the range of $R_{\text {mag }}=17.3-19$. Such magnitude allows for recording of an $\mathrm{H}_{2}$ absorption spectrum of typical SNR $\geq 20$ and resolution of $R=50000$ in an amount of $10 \mathrm{hrs}$ at an 8-10 m class telescope, such as the Keck telescope (with the HIRES spectrograph) and the Very Large Telescope (VLT, with the UVES spectrograph). The quasar J2123-005 is a special case displaying extraordinary high brightness $\left(R_{\text {mag }}=15.83\right)$, which was exploited in observations by both telescopes (Malec et al. 2010; van Weerdenburg et al. 2011). Further, good quality $\mathrm{H}_{2}$ absorption spectra can be obtained for column densities of $\log _{10} N\left(\mathrm{H}_{2}\right)\left[\mathrm{cm}^{-2}\right]=17-18.5$. System Q0347-383 with $\log _{10} N\left(\mathrm{H}_{2}\right)\left[\mathrm{cm}^{-2}\right]=14.5$ produces nevertheless a good quality spectrum with non-saturated lines (Ivanchik et al. 2005; Wendt and Molaro 2012), while system J1237+064 exhibits strongly saturated absorption lines for the highest populated quantum states of $\mathrm{H}_{2}$ (Noterdaeme et al. 2010).

Quasar absorption spectra are treated in a 'comprehensive fitting' analysis (Malec et al. 2010), where all $\mathrm{H}_{2}$ lines are described by a set of molecular parameters, $\lambda_{i}^{0}$ for the restframe wavelength, $f_{i}$ for the line oscillator strength, and $\Gamma_{i}$ for the natural broadening coefficient, and the sensitivity coefficients $K_{i}$ and invoked in the equation governing the comparison between spectra:

$$
\lambda_{i}^{z}=\lambda_{i}^{0}\left(1+z_{\mathrm{abs}}\right)\left(1+K_{i} \frac{\Delta \mu}{\mu}\right) .
$$

The rest frame wavelengths $\lambda_{i}^{0}$ of the $\mathrm{H}_{2}$ absorption lines have been determined to high accuracy in dedicated laboratory experiments using tunable vacuum ultraviolet lasers (Ubachs and Reinhold 2004; Philip et al. 2004), as well as by a combination of spectroscopic studies aimed at determining level energies of excited states in $\mathrm{H}_{2}$ from which the vacuum ultraviolet absorption lines can be calculated at high accuracy (Bailly et al. 2010). Also lines of the HD isotopologue are used in the astronomical analyses for which accurate laboratory data are available (Ivanov et al. 2008). The status of these laboratory experiments is that the transition wavelengths of the $\mathrm{H}_{2}$ absorptions may be considered exact for the purpose of comparison to quasar data recorded with the HIRES spectrometer at Keck and the UVES 


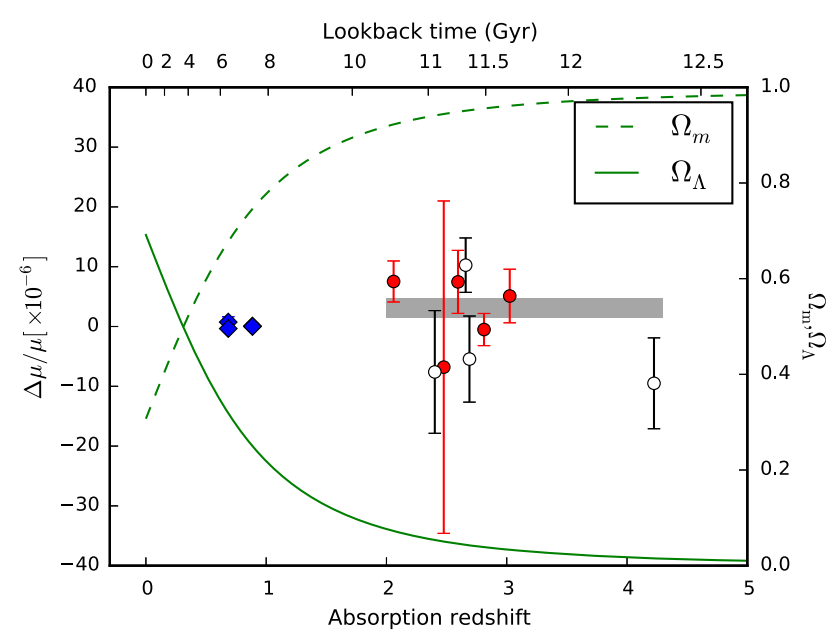

Fig. 1 Values for the relative variation of the proton-to-electron mass ratio $\Delta \mu / \mu$ plotted as a function of redshift and look-back time for nine $\mathrm{H}_{2}$ absorption systems analyzed in detail (Q1232+082 not included). Open circles: data corrected for long-range wavelength distortions; closed (red) circles: uncorrected data. Results are plotted alongside with evolution of cosmological parameters for $\Omega_{m}$ (dashed line) and $\Omega_{\Lambda}$ (full line), with respect to the right-hand vertical axis. The gray bar represents the average for $\Delta \mu / \mu$ with $\pm 1 \sigma$ uncertainty limits from the $\mathrm{H}_{2}$ systems analyzed. For comparison results from radio astronomical observations at lower redshift $(z<1)$ are plotted as well (diamonds-blue). Figure reproduced from Ubachs et al. (2016)

spectrometer at VLT. The values $f_{i}$ and $\Gamma_{i}$ for the absorption lines are obtained from ab initio calculations of the $\mathrm{H}_{2}$ molecule (Abgrall et al. 2000). Values for the sensitivity coefficients $K_{i}$ were derived by a number of groups using independent techniques (Varshalovich and Levshakov 1993; Meshkov et al. 2006; Ubachs et al. 2007).

In the analysis of astronomical spectra each spectral line is folded by a Doppler parameter $b$, shifted by the redshift parameter $1+z$ and its intensity multiplied by the column density $N_{J}$ for each populated quantum state of the $\mathrm{H}_{2}$ molecule. Typically only states with $v=0$ and $J=0-5$ will be populated to the extent that absorption is detected. Hence, from the broadening parameters $\Gamma_{i}$ and $b$, further folded by the instrument function of the spectrograph, a Voigt profile shape for the lines is obtained. While applying the fitting routine to the entire spectrum multiple velocity components, introduced as multiple redshift parameters $z_{i}$, will be allowed for optimizing the entire spectrum. In this analysis the occurrence of Lyman- $\alpha$ forest lines as well as metal line absorption will be included. In the final stage of this comprehensive fitting method, after a well-behaved absorption model is created, $\Delta \mu / \mu$ is introduced as a final free parameter and fitted.

This procedure has been applied to several of the ten absorption systems listed in Table 1, while for some other systems a 'line-by-line' fitting model was used (Thompson et al. 2009; Wendt and Molaro 2012). The latter method exhibits some drawbacks when dealing with overlapped lines in the fit (Malec et al. 2010; King et al. 2011). Results for the values $\Delta \mu / \mu$ obtained from the various systems at redshifts in the range $z=2.0-4.2$ analyzed have been collected in Fig. 1. A weighted average yields $\Delta \mu / \mu=(3.1 \pm 1.6) \times 10^{-6}$. This result, indicating a small $1.9 \sigma$ effect of a varying $\mu$ is, in view of its small significance, treated as a null-result. This null-result may also be phrased as $|\Delta \mu / \mu|<5 \times 10^{-6}$ at a $3 \sigma$ significance level (Ubachs et al. 2016). 


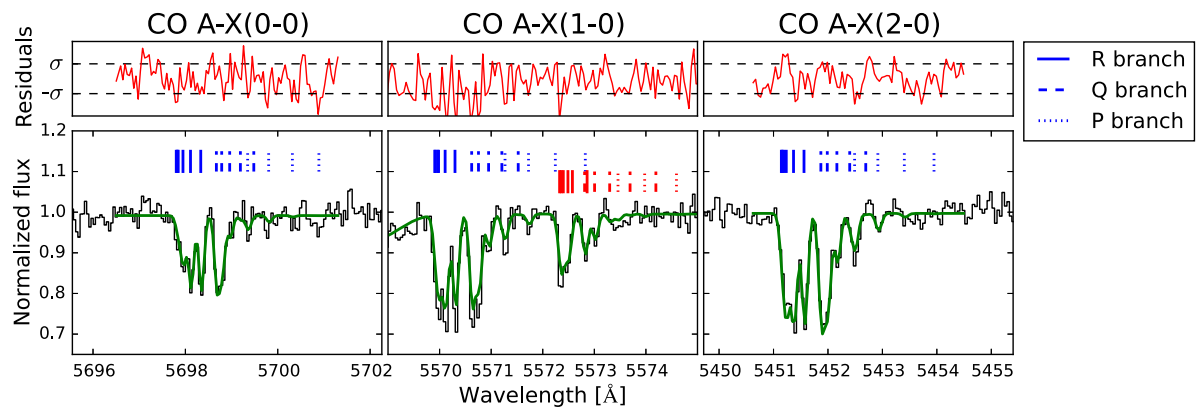

Fig. 2 Spectra of the A-X $(v, 0)$ bands, for $v=0-2$, observed in the line of sight toward $\mathrm{J} 1237+064$ at redshift $z=2.69$. In the upper panels the residuals from a fit are displayed

\section{Optical Detection of the CO Molecule}

Carbon monoxide is the second most abundant gaseous molecule in the Universe and its A-X electronic band system, occurring at rest-frame wavelengths of $\lambda<1544 \AA$, is observed in a number of quasar absorption systems (Noterdaeme et al. 2008, 2010, 2011, 2017). Since the wavelength interval of the A-X bands is longward of the Lyman- $\alpha$ line these CO spectra are not overlaid by Lyman- $\alpha$ forest lines.

In the quasar absorption system $\mathrm{J} 1237+064 \mathrm{CO}$ is detected at $z=2.69$ (with ESO-VLT) with a column density of $\log _{10} N(\mathrm{CO})\left[\mathrm{cm}^{-2}\right]=14.2$ and absorption bands $\mathrm{A}-\mathrm{X}(v, 0)$ for $v=0-8$ were observed in the range $\lambda=5696-4880 \AA$. In addition also the $\mathrm{B}-\mathrm{X}(0,0)$, $\mathrm{C}-\mathrm{X}(0,0)$ and $\mathrm{E}-\mathrm{X}(0,0)$ bands were observed in the bluer part of the spectrum. Molecular parameters were measured in laser-based and synchrotron-based spectroscopic absorption studies (Salumbides et al. 2012) and invoked in the 'comprehensive fitting' method based on Eq. (1). In Fig. 2 spectra of the three lowest bands of CO A-X, as observed in $\mathrm{J} 1237+064$, are displayed with a model fit. The analysis of the CO spectrum yields a value of $\Delta \mu / \mu=(2.3 \pm 1.7) \times 10^{-5}$ (Daprà et al. 2016). This shows that the CO method is somewhat less constraining than the $\mathrm{H}_{2}$ method, which is ascribed to the fact that the details of the $\mathrm{CO}$ absorption features are spread over less pixels in the spectrum than those of $\mathrm{H}_{2}$. The range of $K$-coefficients is similar for both molecules. Recently also $\mu$-variation was analyzed from a second absorber, J0000+0048 at $z=2.52$, resulting in $\Delta \mu / \mu=(1.8 \pm 2.3) \times 10^{-5}$ (Daprá et al. 2017a).

\section{Detection of $\mathrm{H}_{2}$ in the Photosphere of White Dwarfs}

The spectrum of molecular hydrogen can also be used to search for a possible dependence of the fundamental constant $\mu$ on a gravitational field. Theoretically such phenomenon might be explained in 'chameleon' scenarios of varying constants (Khoury and Weltman 2004; Flambaum and Shuryak 2008). Some white dwarfs (WD) exhibit a thin photosphere layer in which molecular hydrogen molecules give rise to an absorption spectrum (Xu et al. 2013). The conditions applying to the $\mathrm{H}_{2}$ molecules in such circumstances are extreme. Since white dwarfs are collapsed stars of typically a solar mass shrunk to a radius $R$ of $1 \%$ of the Sun, the gravitational potential $\phi=G M / R c^{2}$ is about 10000 times that on the Earth's surface. Further, the temperature of the photosphere is typically in excess of $10000 \mathrm{~K}$. Spectra of the photosphere of two white dwarfs, GD-29-38 (or WD2326+049) and GD-133 

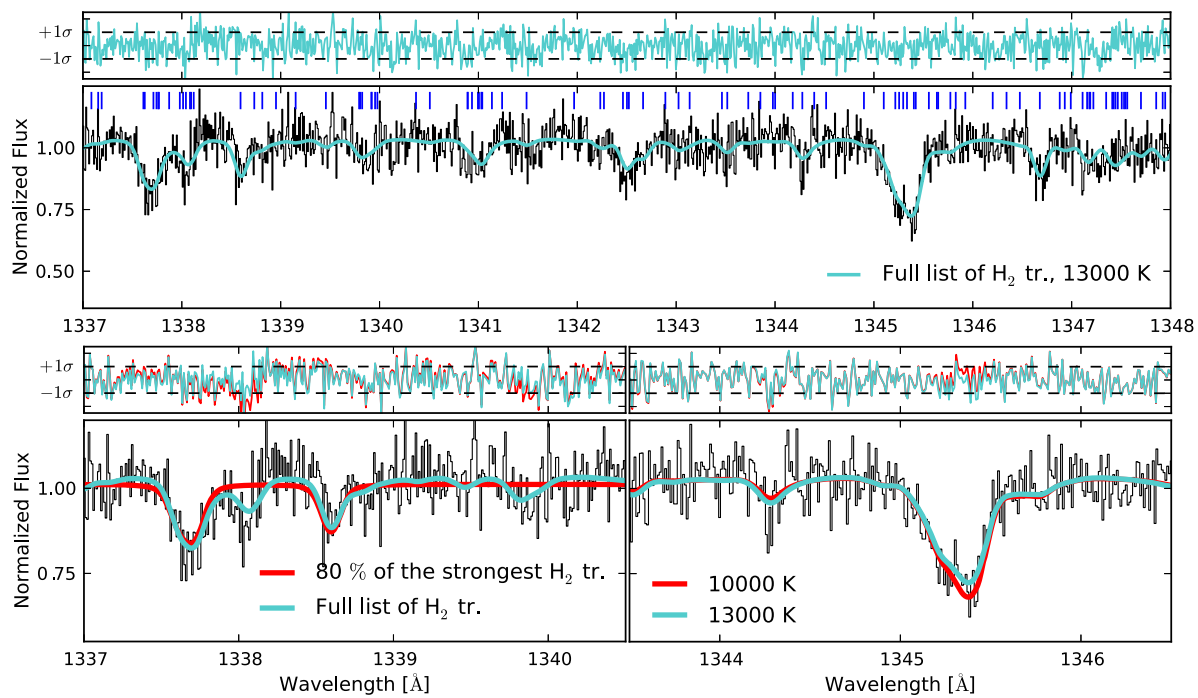

Fig. 3 Part of the absorption spectrum of the photosphere of the white dwarf system GD29-38, overlaid with a model spectrum for temperatures of $T=10000 \mathrm{~K}$ and using the $80 \%$ strongest lines (red line), and a model for $T=13000 \mathrm{~K}$ and the full list of $\mathrm{H}_{2}$ absorption lines (blue-green line). The total spectrum used in the analysis extends from $1310-1412 \AA$

(WD1116+026) were observed with the Cosmic Origins Spectrograph (COS) aboard the Hubble Space Telescope (HST).

Studies aiming to investigate gravitational effects in dim objects as WD stars can preferably be performed on systems observed inside the Galaxy. However, in such case there will be no redshift to shift the absorption profiles into the atmospheric transmission window. Galactic WD spectra should then be recorded in the vacuum ultraviolet range, for which COS-HST is ideally suited. An example of a Galactic WD $\mathrm{H}_{2}$ absorption spectrum is displayed in Fig. 3 for the case of GD-29-38. In view of the high local temperatures higher vibrational states of $\mathrm{H}_{2}$ are populated in the WD photospheres, in contrast to the cold environments probed in quasar absorption, where only $\mathrm{H}_{2}(v=0)$ is detected. In view of the Franck-Condon factors of $\mathrm{H}_{2}$ the Lyman bands B-X $(1,3)$ and $(1,4)$ are the most strongly represented in the observed spectrum which covers the range 1310-1412 A (Bagdonaite et al. 2014a). At temperatures of $T>10000 \mathrm{~K}$ rotational quantum states of up to $J=25$ are effectively populated and a simulation of the spectra involves starting from the partition function including all states populated (Salumbides et al. 2015):

$$
P_{v, J}(T)=\frac{g_{n}(2 J+1) e^{\frac{-E_{v, J}}{k T}}}{\sum_{v=0}^{v_{\max }} \sum_{J=0}^{J_{\max }(v)} g_{n}(2 J+1) e^{\frac{-E_{v, J}}{k T}}}
$$

Based on this partition function and assuming thermodynamic equilibrium for the $\mathrm{H}_{2}$ populated quantum states a fit can be made to the observed spectra, with temperature $T$ and $\Delta \mu / \mu$ as free parameters, again using Eq. (1) for the absorption model. This method results in constraints on dependences of $\mu$ on a gravitational field quantified as $\Delta \mu / \mu=$ $(-5.8 \pm 3.9) \times 10^{-5}$ for GD-29-38 (at $\left.\phi=1.9 \times 10^{-4}\right)$ and $\Delta \mu / \mu=(-2.7 \pm 4.9) \times 10^{-5}$ for GD-133 (at $\phi=1.2 \times 10^{-4}$ ) (Bagdonaite et al. 2014a). Thus far the objects GD-29-38 
Table 2 List of extra-galactic radio sources with molecules detected in absorption searching for varying constants

\begin{tabular}{lllll}
\hline Radio Quasar & $z_{\text {abs }}$ & RA(J2000) & Decl.(J2000) & Refs. \\
\hline PKS1830-211 & 0.882 & $18: 33: 39.9$ & $-21: 03: 40$ & $\begin{array}{l}\text { Henkel et al. (2009), } \\
\text { Bagdonaite et al. (2013a, 2013b), } \\
\text { Kanekar et al. (2015) } \\
\text { B0218+357 }\end{array}$ \\
$\begin{array}{llll}\text { Murphy et al. (2008), } \\
\text { Kanekar (2011) }\end{array}$ & & $\begin{array}{l}\text { Darling (2004) } \\
\text { Kanekar et al. (2005) }\end{array}$ \\
\hline
\end{tabular}

and GD-133 are the only white dwarf stars for which an $\mathrm{H}_{2}$ absorption spectrum of sufficient SNR was obtained. In a third system, GD-31 (Xu et al. 2013), $\mathrm{H}_{2}$ was observed but the spectrum is of too low quality to derive a competitive constraint on $\mu$.

\section{Radio Astronomical Observation of Polyatomic Molecules}

Radio astronomy is the preferred technique for identifying molecules in the interstellar medium and currently well over a 100 molecular species have been detected through observations in our Galaxy. Only a limited sample of extragalactic radio sources at intermediate redshift $(0.2<z<1)$ with molecular absorption in its sightline have been identified, the four listed in Table 2 which have been used to search for varying constants on a cosmological time scale, and a fifth system B1504+377 in which $\mathrm{OH}$ has been detected (Kanekar and Chengalur 2002). The radio-loud quasars B0218+357 and PKS 1830-211 are strongly lensed radio galaxies with the radio source far in the background, exhibit a large extinction, $A_{v} \sim 10-100$, and thus a very high molecular fraction.

Most of the molecules detected in the radio range are observed through pure rotational lines in the spectrum, for which the sensitivity coefficients are $K_{\mu}=-1$ (Jansen et al. 2014). This implies that all such transitions exhibit the same sensitivity to $\mu$-variation. Hence $\mu$-variation does not produce a differential effect, to be distinguished from a cosmological or a Doppler shift, not even if transitions of different molecules are included in a combined analysis. If however molecules are considered that exhibit other internal modes of energy a favorable situation arises. The ammonia molecule exhibits an inversion splitting associated with a tunneling inversion mode (the $\mathrm{N}$-atom tunneling through the plane of the three $\mathrm{H}$-atoms) which is very sensitive to a mass variation, in view of quantum tunneling being exponentially dependent on mass. This gives rise to a sensitivity coefficient of $K_{\mu}=-4.2$ for the typical $21 \mathrm{GHz}$ lines of $\mathrm{NH}_{3}$ (Flambaum and Kozlov 2007). However, again the various rotational-tunneling lines all exhibit the same $K$-value, so that purely rotational lines of other molecules (at $K_{\mu}=-1$ ) must be included to derive constraints on $\Delta \mu / \mu$. Typically $\mathrm{HC}_{3} \mathrm{~N}$ lines can be used for that purpose (Henkel et al. 2009), but the analysis then is based on the assumption that $\mathrm{NH}_{3}$ and $\mathrm{HC}_{3} \mathrm{~N}$ molecules reside in the same location in the extragalactic absorbing clouds. Ammonia has thus far been observed in two intermediate redshift objects, PKS1830-211 (Henkel et al. 2009) and B0218+357 (Murphy et al. 2008).

The methanol molecule is an exemplary case where these shortcomings are circumvented. The motion of hindered internal rotation of the $\mathrm{OH}$-group over the $\mathrm{CH}_{3}$ group gives rise to a tunneling motion for which some rotational-torsional transitions exhibit very large $K_{\mu}$ coefficients, with values of $K_{\mu}=-7.4$ and $K_{\mu}=-33$ (Jansen et al. 2011; Levshakov 
Fig. 4 The four methanol absorption lines observed with the Effelsberg radio telescope at $z=0.88582$ towards the radio-loud quasar PKS1830-211. Figure adapted from Bagdonaite et al. (2013b)

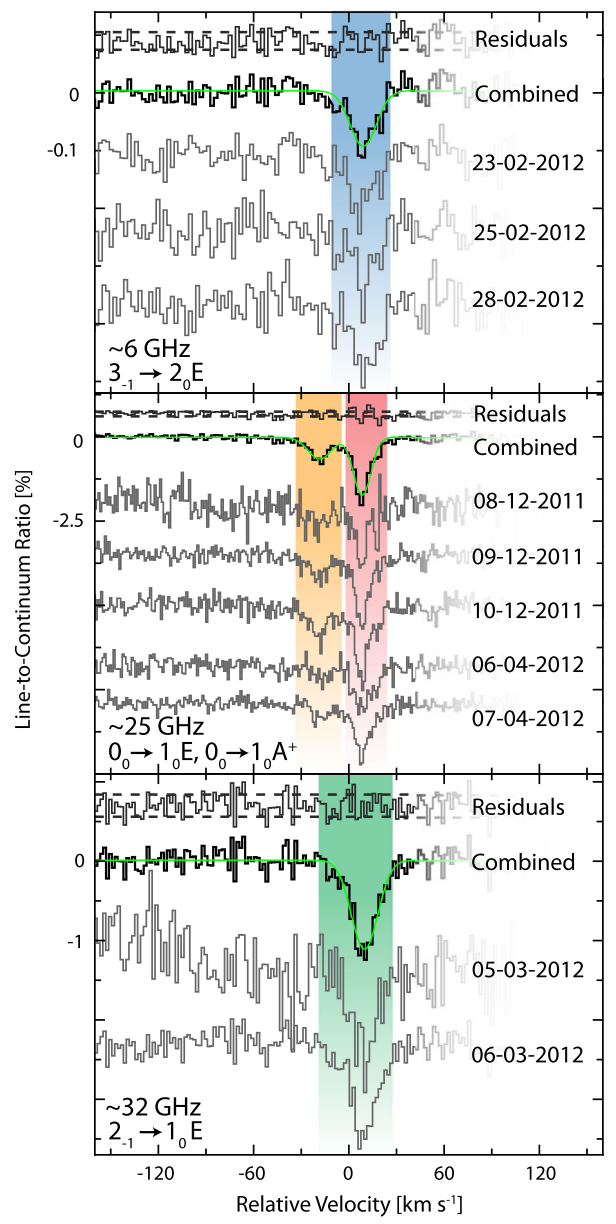

et al. 2011). When these transitions are combined with pure rotational transitions, of the same molecular species, then a very sensitive analysis of $\mu$-variation can be performed. This was done in an observation of four radio lines in the methanol spectrum, ranging from $6.5 \mathrm{GHz}$ to $32 \mathrm{GHz}$, with the Effelsberg $100 \mathrm{~m}$ dish radio telescope (see Fig. 4; Bagdonaite et al. 2013b). After that initial study more extended observations of methanol in the same object have been observed, using also the IRAM 30 m telescope and the ALMA telescope array (Bagdonaite et al. 2013a), as well as the Extended Very Large Array (Kanekar et al. 2015). These studies have produced the tightest constraint of $|\Delta \mu / \mu|<10^{-7}$, however only for a single object and at a single redshift, equivalent to 7.5 Gyrs look-back time or half the age of the Universe. The stringent constraints on $\Delta \mu / \mu$ derived from $\mathrm{NH}_{3}$ and $\mathrm{CH}_{3} \mathrm{OH}$ at redshifts $z=0.68$ and $z=0.88$ are included in Fig. 1.

In two other radio systems PMN J0134-0931 with a lensed galaxy at $z=0.765$, and PKS1413+135 absorption of $\mathrm{OH}$ radicals was analyzed that provided combined constraints on $\mu$ and $\alpha$. The observation of $\mathrm{OH}$ lines at $18 \mathrm{~cm}$ was combined with that of the $\mathrm{HI} 21 \mathrm{~cm}$ line, from which contributions to varying $\mu$ and $\alpha$ may be disentangled (Darling 2004; Kanekar et al. 2005). 
Most investigations on varying constants focus on absorption, because cold environments may be probed without too much turbulent motion and line broadening. It was shown however, that emission of $\mathrm{CO}$ combined with emission of atomic C I, towards the lensed galaxy HLSJ091828.6 +514223 at $z=5.2$ could be employed to derive a constraint on $\mu$-variation at such high redshifts (Levshakov et al. 2012).

\section{Future Opportunities and Instrumentation}

The accuracy of the $\mathrm{H}_{2}$ and $\mathrm{CO}$ optical absorption methods as discussed in Sects. 2 and 3 is limited by the signal-to-noise ratio (SNR) obtained in the spectra recorded at the 8-10 m class telescopes. Extending the integration time will improve the spectra and therewith the statistical constraint on $\Delta \mu / \mu$, but the SNR scales only with the root of measurement time. Since for the measurement of the $\mathrm{H}_{2}$ absorption spectrum of Q2348-011 already an averaging time of $19 \mathrm{hrs}$ on target was applied (Bagdonaite et al. 2012), it seems unrealistic to further extend such long observation periods on 8-10 m class telescopes. However, the construction of a next generation optical telescopes with 30-40 m dishes, such as the European Extremely Large Telescope, the Thirty Meter Telescope and the Giant Magellan Telescope, will deliver the prospect of increased photon collection and drastically improved signal-tonoise ratio. This will straightforwardly lead to tighter bounds on $\Delta \mu / \mu$ with the $\mathrm{H}_{2}$ and CO optical methods.

The spectrographs used for $\mu$-variation studies (UVES at VLT, and HIRES at Keck) are designed such that a resolving power of $R \sim 50000$ is obtained for an entrance slit corresponding to 0.8 arcseconds; this value matches 'regular-to-good' seeing conditions so that all light can be captured within the slit at seeing conditions of $0.8^{\prime \prime}$. For the molecular hydrogen absorbing systems investigated so far it was found that the Doppler broadening of the individual $\mathrm{H}_{2}$ features in all of the cases yielded superthermal line broadenings in excess of the 50000 resolving power limit, most likely due to turbulent motion inside the absorbing galaxy or unresolved velocity substructures. In the specific case of J2123-005 spectra were measured at $R=55000$ with UVES-VLT (van Weerdenburg et al. 2011) and $R=110000$ with HIRES-Keck (Malec et al. 2010), but the linewidths in the observed spectra were the same, hence fully governed by superthermal Doppler broadening. In such cases, for all systems investigated (see Table 1), the effective Doppler width greatly exceeds the kinematic width that would correspond to the Boltzmann population temperatures, which are for $\mathrm{H}_{2}$ typically 50-150 K (Petitjean et al. 2002; Srianand et al. 2005). Although it might be possible that an $\mathrm{H}_{2}$ absorbing system will be found, exhibiting no turbulent motions and a strongly decreased Doppler broadening, as of now it appears that improved resolution beyond 50000 would probably not benefit the $\mathrm{H}_{2}$ method. The planned installation of the ESPRESSO spectrograph at ESO-VLT with its high-resolution mode of $R=120000$ and its very-high-resolution mode of $R=220000$ would provide an opportunity to further test this hypothesis.

Wavelength calibration is a crucial issue in a spectroscopic search for varying constants, because the methods rely on exploring the smallest differential wavelength shifts between molecular absorption lines. The spectra are generally calibrated against a ThAr reference spectrum measured immediately after the quasar exposures without resetting its geometry. It was found that "intra-order" distortions occur in the echelle-grating based measurements (Griest et al. 2010; Whitmore et al. 2010). In addition, it was found that long-range wavelength distortions also affect the calibration of the quasar absorption spectra when calibrated against the ThAr lines. This is probably due to the fact that the light beams as originating 
from far-distant objects and the light beams from the ThAr cell on the telescope platform travel along different paths (Rahmani et al. 2013; Whitmore and Murphy 2015). The resulting distortions of the wavelength scale may mimic a $\Delta \mu / \mu$ effect. For analyzing these miscalibration phenomena exposures of either solar twin stars or of asteroids are used, which may be compared with an accurate high-resolution Fourier-transform spectra measured of the Sun (Molaro et al. 2008). Such recalibration methods may be applied to assess a value for the systematic uncertainty in the determination of $\Delta \mu / \mu$, or even counter-distort the wavelength scale to obtain a fiducial value of a constraint on $\Delta \mu / \mu$, as was pursued in some studies (Rahmani et al. 2013; Bagdonaite et al. 2014b; Daprà et al. 2015).

Some of the problems of wavelength calibration of astronomical spectra can be resolved by fiber-feeding the light into the spectrograph. Already for the ESPRESSO spectrograph the light from the VLT large-dish will be collected into a multi-mode fiber before entering the spectrograph, with the goal to discriminate against angular differences between light paths. A problem with fiber-feeding is the additional loss of photons, in particular in the UV part of the spectrum. In fact the fiber-fed spectrographs will not be specified for the range 3000-3800 $\AA$, a range that contains the central information for the $\mathrm{H}_{2}$ method in case of quasars at redshifts $z=2-3$. Hence fiber feeding the spectrograph will be detrimental in application to most of the $\mathrm{H}_{2}$ absorbers that fall in that redshift range (see Table 1).

Another improvement, currently established on some telescope-based spectrographs, is the use of frequency comb lasers at the observatories (Wilken et al. 2012). Frequency comb lasers deliver a very dense spectrum of frequency markers at a separation exactly governed by an atomic clock, thus constituting an ideal reference spectrum. However, extension of the comb markers to the UV part of the spectrum at 3000-3800 $\AA$ is not obvious. Again, a combination with fiber-feeding is anticipated, resulting in similar problems for the $\mathrm{H}_{2}$ method as mentioned above.

The method to detect a dependence of $\mu$ on gravitational fields, as discussed in Sect. 4 , requires a high resolution satellite-based VUV spectrograph, since zero-redshift absorption by $\mathrm{H}_{2}$ occurs in the wavelength range for which the Earth's atmosphere is opaque. Currently the COS-spectrograph aboard the HST serves this purpose although for the study of $\mathrm{H}_{2}$ in the Milky Way it would be desirable to use a spectrometer of a resolving power beyond that of COS $(R=20000)$. Repair on HST instruments is likely to stop after the launch of the James Webb Space Telescope in the near future. The latter takes advantage of positioning in a Lagrange point of the Earth orbit around the sun at 1.5 million km from Earth, but is only equipped with instruments for the infrared. While in the past Galactic molecular hydrogen was first detected from a rocket-sounding mission (Carruthers 1970), thereafter dedicated VUV-spectrographs were launched in orbit: the Copernicus mission, the Orpheus telescope aboard a Space Shuttle, the Far Ultraviolet Spectroscopic Explorer (FUSE) mission, and the Space Telescope Imaging Spectrograph (STIS) and the Cosmic Origins Spectrograph (COS) instruments aboard the HST. The possibility of detecting and studying $\mathrm{H}_{2}$ within the Milky Way, for any astrophysical purpose, is strongly endangered after HST and its instruments reach the end of their lifetime. A possible follow-up mission is that of the Large/UV/Optical/Infrared (LUVOIR) surveyor covering the $\mathrm{H}_{2}$ absorption wavelength range, which is however still in a preparation phase (France et al. 2016).

As for the radio astronomical observations in search for a varying $\mu$, discussed in Sect. 5 , it is established that probing the methanol molecule has two distinct advantages: $\mathrm{CH}_{3} \mathrm{OH}$ exhibits the most sensitive transitions and it exhibits transitions with a wide variety of sensitivities, such that there is no need to compare with other molecular species providing anchor lines. However, thusfar only a single absorbing system with methanol toward a radio-loud quasar is found. The object at $z=0.88$ in the line-of-sight toward PKS1830-211 is a lensed 
galaxy, while the background source is a blazar and exhibiting a variable luminosity (Muller et al. 2011), which brings some difficulties when studying it in the context of $\mu$-variation (Bagdonaite et al. 2013a). In a strategy of tightening the limitations on $\Delta \mu / \mu$ the most sensitive radio observatories could be used for finding additional methanol absorbers, preferably at higher redshifts $(z>1)$. For such survey studies the Atacama Large Millimeter Array (ALMA) observatory could be used, targeting high frequency transitions of methanol in the ALMA window $(>100 \mathrm{GHz}$ ). It should be noted however that the roto-torsional modes in methanol, exhibiting the largest sensitivity coefficients, are at lower frequencies, typically at $<50 \mathrm{GHz}$, depending on redshift. While currently the Effelsberg $100 \mathrm{~m}$ telescope (Bagdonaite et al. 2013b) and the Extended Very Large Array (Kanekar et al. 2015) are suitable observatories for the low-frequency range, in future the Square Kilometer Array (SKA) will be a very sensitive instrument to probe sensitive transitions in methanol for further constraining fundamental constants as the proton-electron mass ratio, or find a positive effect of new physics beyond the prevailing Standard Model.

Acknowledgements The author acknowledges financial support from the European Research Council (ERC) under the European Union's Horizon 2020 research and innovation programme (Grant Agreement No. 670168).

Open Access This article is distributed under the terms of the Creative Commons Attribution 4.0 International License (http://creativecommons.org/licenses/by/4.0/), which permits unrestricted use, distribution, and reproduction in any medium, provided you give appropriate credit to the original author(s) and the source, provide a link to the Creative Commons license, and indicate if changes were made.

\section{References}

H. Abgrall, E. Roueff, I. Drira, Total transition probability and spontaneous radiative dissociation of B, C, B' and D states of molecular hydrogen. Astron. Astrophys. Suppl. Ser. 141, 297-300 (2000)

D. Albornoz Vásquez, H. Rahmani, P. Noterdaeme, P. Petitjean, R. Srianand, C. Ledoux, Molecular hydrogen in the $z_{a b s}=2.66$ damped Lyman- $\alpha$ absorber towards QJ0643-5041. Physical conditions and limits on the cosmological variation of the proton-to-electron mass ratio. Astron. Astrophys. 562, 88 (2014)

J. Bagdonaite, M.T. Murphy, L. Kaper, W. Ubachs, Constraint on a variation of the proton-to-electron mass ratio from $\mathrm{H}_{2}$ absorption towards quasar Q2348-011. Mon. Not. R. Astron. Soc. 421, 419-425 (2012)

J. Bagdonaite, M. Daprà, P. Jansen, H.L. Bethlem, W. Ubachs, S. Muller, C. Henkel, K.M. Menten, Robust constraint on a drifting proton-to-electron mass ratio at $z=0.89$ from methanol observation at three radio telescopes. Phys. Rev. Lett. 111, 231101 (2013a)

J. Bagdonaite, P. Jansen, C. Henkel, H.L. Bethlem, K.M. Menten, W. Ubachs, A stringent limit on a drifting proton-to-electron mass ratio from alcohol in the early Universe. Science 339, 46-48 (2013b)

J. Bagdonaite, E.J. Salumbides, S.P. Preval, M.A. Barstow, J.D. Barrow, M.T. Murphy, W. Ubachs, Limits on a gravitational field dependence of the proton-electron mass ratio from $\mathrm{H}_{2}$ in white dwarf stars. Phys. Rev. Lett. 113, 123002 (2014a)

J. Bagdonaite, W. Ubachs, M.T. Murphy, J.B. Whitmore, Analysis of molecular hydrogen absorption toward QSO B0642-5038 for a varying proton-to-electron mass ratio. Astrophys. J. 782, 10 (2014b)

J. Bagdonaite, W. Ubachs, M.T. Murphy, J.B. Whitmore, Constraint on a varying proton-electron mass ratio 1.5 billion years after the Big Bang. Phys. Rev. Lett. 114, 071301 (2015)

J.N. Bahcall, M. Schmidt, Does the fine-structure constant vary with cosmic time? Phys. Rev. Lett. 19, 1294 (1967)

D. Bailly, E.J. Salumbides, M. Vervloet, W. Ubachs, Accurate level energies in the $E F^{1} \Sigma_{g}^{+}, G K^{1} \Sigma_{g}^{+}$, $H^{1} \Sigma_{g}^{+}, B^{1} \Sigma_{u}^{+}, C^{1} \Pi_{u}, B^{\prime 1} \Sigma_{u}^{+}, D^{1} \Pi_{u}, I^{1} \Pi_{g}, J^{1} \Delta_{g}$ states of $\mathrm{H}_{2}$. Mol. Phys. 108, 827-846 (2010)

S.A. Balashev, V.V. Klimenko, A.V. Ivanchik, D.A. Varshalovich, P. Petitjean, P. Noterdaeme, Molecular hydrogen absorption systems in Sloan digital sky survey. Mon. Not. R. Astron. Soc. 440, 225-239 (2014)

J. Barrow, H. Sandvik, J. Magueijo, Behavior of varying- $\alpha$ cosmologies. Phys. Rev. D 65, 063504 (2002)

$\mathrm{X}$. Calmet, H. Fritzsch, The cosmological evolution of the nucleon mass and the electroweak coupling constants. Eur. Phys. J. C 24, 639-642 (2002) 
G.R. Carruthers, Rocket observation of interstellar molecular hydrogen. Astrophys. J. Lett. 161, 81 (1970)

M. Daprà, J. Bagdonaite, M.T. Murphy, W. Ubachs, Constraint on a varying proton-to-electron mass ratio from molecular hydrogen absorption toward quasar SDSS J123714.60+064759.5. Mon. Not. R. Astron. Soc. 454, 489-506 (2015)

M. Daprà, M.L. Niu, E.J. Salumbides, M.T. Murphy, W. Ubachs, Constraint on a cosmological variation in the proton-to-electron mass ratio from electronic CO absorption. Astrophys. J. 826, 192 (2016)

M. Daprá, P. Noterdaeme, M. Vonk, M.T. Murphy, W. Ubachs, Analysis of carbon monoxide absorption at $z_{a b s}=2.5$ to constrain variation of the proton-to-electron mass ratio. Mon. Not. R. Astron. Soc. 467, 3848-3855 (2017a)

M. Daprá, M. van der Laan, M.T. Murphy, W. Ubachs, Constraint on a varying proton-to-electron mass ratio from $\mathrm{H}_{2}$ and $\mathrm{HD}$ absorption at $z_{a b s}=2.34$. Mon. Not. R. Astron. Soc. 465, 4057 (2017b)

J. Darling, A laboratory for constraining cosmic evolution of the fine-structure constant: Conjugate 18 centimeter OH lines toward PKS $1413+135$ at $z=0.24671$. Astrophys. J. 612, 58 (2004)

V. Dumont, J.K. Webb, Modelling long-range wavelength distortions in quasar absorption echelle spectra. Mon. Not. R. Astron. Soc. 468, 1568-1574 (2017)

V.A. Dzuba, V.V. Flambaum, J.K. Webb, Space-time variation of physical constants and relativistic corrections in atoms. Phys. Rev. Lett. 82, 888-891 (1999)

V.V. Flambaum, M.G. Kozlov, Limit on the cosmological variation of $\mathrm{m}_{p} / \mathrm{m}_{e}$ from the inversion spectrum of ammonia. Phys. Rev. Lett. 98, 240801 (2007)

V.V. Flambaum, E.V. Shuryak, How changing physical constants and violation of local position invariance may occur?, in Nuclei and Mesoscopic Physics, ed. by P. Danielewicz, P. Piecuch, V. Zelevinsky. AIP Conference Series, vol. 995 (2008), pp. 1-11

K.C. France, B.T. Fleming, K. Hoadley, CHISL: The combined high-resolution and imaging spectrograph for the LUVOIR surveyor. J. Astron. Telesc. Instrum. Syst. 2, 041203 (2016)

K. Griest, J.B. Whitmore, A.M. Wolfe, J.X. Prochaska, J.C. Howk, G.W. Marcy, Wavelength accuracy of the Keck HIRES spectrograph and measuring changes in the fine structure constant. Astrophys. J. 708, 158-170 (2010)

C. Henkel, K.M. Menten, M.T. Murphy, N. Jethava, V.V. Flambaum, J.A. Braatz, S. Muller, J. Ott, R.Q. Mao, The density, the cosmic microwave background, and the proton-to-electron mass ratio in a cloud at redshift 0.9. Astron. Astrophys. 500, 725-734 (2009)

A. Ivanchik, P. Petitjean, D. Varshalovich, B. Aracil, R. Srianand, H. Chand, C. Ledoux, P. Boissé, A new constraint on the time dependence of the proton-to-electron mass ratio. Analysis of the Q0347-383 and Q0405-443 spectra. Astron. Astrophys. 440, 45-52 (2005)

A.V. Ivanchik, P. Petitjean, S.A. Balashev, R. Srianand, D.A. Varshalovich, C. Ledoux, P. Noterdaeme, HD molecules at high redshift: The absorption system at $z=2.3377$ towards Q1232+082. Mon. Not. R. Astron. Soc. 404, 1583-1590 (2010)

T.I. Ivanov, M. Roudjane, M.O. Vieitez, C.A. de Lange, W.-Ü.L. Tchang-Brillet, W. Ubachs, HD as a probe for detecting mass variation on a cosmological time scale. Phys. Rev. Lett. 100, 093007 (2008)

P. Jansen, L.-H. Xu, I. Kleiner, W. Ubachs, H.L. Bethlem, Methanol as a sensitive probe for spatial and temporal variations of the proton-to-electron mass ratio. Phys. Rev. Lett. 106, 100801 (2011)

P. Jansen, H.L. Bethlem, W. Ubachs, Perspective: Tipping the scales: Search for drifting constants from molecular spectra. J. Chem. Phys. 140, 010901 (2014)

N. Kanekar, Constraining changes in the proton-electron mass ratio with inversion and rotational lines. Astrophys. J. Lett. 728, 12 (2011)

N. Kanekar, J.N. Chengalur, Molecular gas at intermediate redshifts. Astron. Astrophys. 381, 73-76 (2002)

N. Kanekar, C.L. Carilli, G.I. Langston, G. Rocha, F. Combes, R. Subrahmanyan, J.T. Stocke, K.M. Menten, F.H. Briggs, T. Wiklind, Constraints on changes in fundamental constants from a cosmologically distant $\mathrm{OH}$ absorber or emitter. Phys. Rev. Lett. 95, 261301 (2005)

N. Kanekar, W. Ubachs, K.M. Menten, J. Bagdonaite, A. Brunthaler, C. Henkel, S. Muller, H.L. Bethlem, M. Daprà, Constraints on changes in the proton-electron mass ratio using methanol lines. Mon. Not. R. Astron. Soc. 448, 104-108 (2015)

J. Khoury, A. Weltman, Chameleon fields: Awaiting surprises for tests of gravity in space. Phys. Rev. Lett. 93, 171104 (2004)

J.A. King, J.K. Webb, M.T. Murphy, R.F. Carswell, Stringent null constraint on cosmological evolution of the proton-to-electron mass ratio. Phys. Rev. Lett. 101, 251304 (2008)

J.A. King, M.T. Murphy, W. Ubachs, J.K. Webb, New constraint on cosmological variation of the proton-toelectron mass ratio from Q0528-250. Mon. Not. R. Astron. Soc. 417, 3010-3024 (2011)

M.G. Kozlov, S.A. Levshakov, Microwave and submillimeter molecular transitions and their dependence on fundamental constants. Ann. Phys. 525, 452-471 (2013)

P. Langacker, G. Segré, M.J. Strassler, Implications of gauge unification for time variation of the fine structure constant. Phys. Lett. B 528, 121-128 (2002) 
S.A. Levshakov, M.G. Kozlov, D. Reimers, Methanol as a tracer of fundamental constants. Astrophys. J. 738, $26(2011)$

S.A. Levshakov, F. Combes, F. Boone, I.I. Agafonova, D. Reimers, M.G. Kozlov, An upper limit to the variation in the fundamental constants at redshift $z=5.2$. Astron. Astrophys. 540, 9 (2012)

A.L. Malec, R. Buning, M.T. Murphy, N. Milutinovic, S.L. Ellison, J.X. Prochaska, L. Kaper, J. Tumlinson, R.F. Carswell, W. Ubachs, Keck telescope constraint on cosmological variation of the proton-to-electron mass ratio. Mon. Not. R. Astron. Soc. 403, 1541-1555 (2010)

V.V. Meshkov, A.V. Stolyarov, A.V. Ivanchik, D.A. Varshalovich, Ab initio nonadiabatic calculation of the sensitivity coefficients for the $X^{1} \Sigma_{g}^{+} \rightarrow B^{1} \Sigma_{u}^{+} ; C^{1} \Pi_{u}$ lines of $\mathrm{H}_{2}$ to the proton-to-electron mass ratio. JETP Lett. 83, 303-307 (2006)

P. Molaro, S.A. Levshakov, S. Monai, M. Centurión, P. Bonifacio, S. D’Odorico, L. Monaco, UVES radial velocity accuracy from asteroid observations. I. Implications for fine structure constant variability. Astron. Astrophys. 481, 559-569 (2008)

S. Muller, A. Beelen, M. Guélin, S. Aalto, J.H. Black, F. Combes, S.J. Curran, P. Theule, S.N. Longmore, Molecules at $z=0.89$. A 4-mm-rest-frame absorption-line survey toward PKS 1830-211. Astron. Astrophys. 535, 103 (2011)

M.T. Murphy, V.V. Flambaum, S. Muller, C. Henkel, Strong limit on a variable proton-to-electron mass ratio from molecules in the distant universe. Science 320, 1611-1613 (2008)

P. Noterdaeme, C. Ledoux, P. Petitjean, R. Srianand, Molecular hydrogen in high-redshift damped Lyman- $\alpha$ systems: The VLT/UVES database. Astron. Astrophys. 481, 327-336 (2008)

P. Noterdaeme, P. Petitjean, C. Ledoux, S. López, R. Srianand, S.D. Vergani, A translucent interstellar cloud at $z=2.69$. CO, $\mathrm{H}_{2}$, and $\mathrm{HD}$ in the line-of-sight to SDSS J123714.60+064759.5. Astron. Astrophys. 523, 80 (2010)

P. Noterdaeme, P. Petitjean, R. Srianand, C. Ledoux, S. López, The evolution of the cosmic microwave background temperature. Measurements of $\mathrm{T}_{\mathrm{CMB}}$ at high redshift from carbon monoxide excitation. Astron. Astrophys. 526, 7 (2011)

P. Noterdaeme, J.-K. Krogager, S. Balashev, J. Ge, N. Gupta, T. Krühler, C. Ledoux, M.T. Murphy, I. Pâris, P. Petitjean, H. Rahmani, R. Srianand, W. Ubachs, Discovery of a Perseus-like cloud in the early Universe-HI-to- $\mathrm{H}_{2}$ transition, carbon monoxide and small dust grains at $z_{a b s} \approx 2.53$ towards the quasar J0000+0048. Astron. Astrophys. 597, 82 (2017)

P. Petitjean, R. Srianand, C. Ledoux, Molecular hydrogen at $z_{a b s}=1.973$ toward Q0013-004: Dust depletion pattern in damped Lyman $\alpha$ systems. Mon. Not. R. Astron. Soc. 332, 383-391 (2002)

J. Philip, J.P. Sprengers, T. Pielage, C.A. de Lange, W. Ubachs, E. Reinhold, Highly accurate transition frequencies in the $\mathrm{H}_{2}$ Lyman and Werner absorption bands. Can. J. Chem. 82, 713-722 (2004)

H. Rahmani, M. Wendt, R. Srianand, P. Noterdaeme, P. Petitjean, P. Molaro, J.B. Whitmore, M.T. Murphy, M. Centurion, H. Fathivavsari, S. D’Odorico, T.M. Evans, S.A. Levshakov, S. Lopez, C.J.A.P. Martins, D. Reimers, G. Vladilo, The UVES large program for testing fundamental physics-II. Constraints on a change in $\mu$ towards quasar HE 0027-1836. Mon. Not. R. Astron. Soc. 435, 861-878 (2013)

M. Rauch, The Lyman alpha forest in the spectra of quasistellar objects. Annu. Rev. Astron. Astrophys. 36, 267-316 (1998)

E. Reinhold, R. Buning, U. Hollenstein, A. Ivanchik, P. Petitjean, W. Ubachs, Indication of a cosmological variation of the proton-electron mass ratio based on laboratory measurement and reanalysis of $\mathrm{H}_{2}$ spectra. Phys. Rev. Lett. 96, 151101 (2006)

E.J. Salumbides, M.L. Niu, J. Bagdonaite, N. de Oliveira, D. Joyeux, L. Nahon, W. Ubachs, CO A-X system for constraining cosmological drift of the proton-electron mass ratio. Phys. Rev. A 86, 022510 (2012)

E.J. Salumbides, J. Bagdonaite, H. Abgrall, E. Roueff, W. Ubachs, $\mathrm{H}_{2}$ Lyman and Werner band lines and their sensitivity for a variation of the proton-electron mass ratio in the gravitational potential of white dwarfs. Mon. Not. R. Astron. Soc. 450, 1237-1245 (2015)

M.P. Savedoff, Physical constants in extra-galactic nebulæ. Nature 178, 688-689 (1956)

R. Srianand, P. Petitjean, C. Ledoux, G. Ferland, G. Shaw, The VLT-UVES survey for molecular hydrogen in high-redshift damped Lyman $\alpha$ systems: Physical conditions in the neutral gas. Mon. Not. R. Astron. Soc. 362, 549-568 (2005)

R.I. Thompson, J. Bechtold, J.H. Black, D. Eisenstein, X. Fan, R.C. Kennicutt, C. Martins, J.X. Prochaska, Y.L. Shirley, An observational determination of the proton-to-electron mass ratio in the early universe. Astrophys. J. 703, 1648-1662 (2009)

W. Ubachs, E. Reinhold, Highly accurate $\mathrm{H}_{2}$ Lyman and Werner band laboratory measurements and an improved constraint on a cosmological variation of the proton-to-electron mass ratio. Phys. Rev. Lett. 92, 101302 (2004)

W. Ubachs, R. Buning, K.S.E. Eikema, E. Reinhold, On a possible variation of the proton-to-electron mass ratio: $\mathrm{H}_{2}$ spectra in the line of sight of high-redshift quasars and in the laboratory. J. Mol. Spectrosc. 241, 155-179 (2007) 
W. Ubachs, J. Bagdonaite, E.J. Salumbides, M.T. Murphy, L. Kaper, Colloquium: Search for a drifting protonelectron mass ratio from $\mathrm{H}_{2}$. Rev. Mod. Phys. 88, 021003 (2016)

J.-P. Uzan, Varying constants, gravitation and cosmology. Living Rev. Relativ. 14, 2 (2011)

F. van Weerdenburg, M.T. Murphy, A.L. Malec, L. Kaper, W. Ubachs, First constraint on cosmological variation of the proton-to-electron mass ratio from two independent telescopes. Phys. Rev. Lett. 106, 180802 (2011)

D.A. Varshalovich, S.A. Levshakov, On a time dependence of physical constants JETP Lett. 58, 237-240 (1993)

D.A. Varshalovich, A.V. Ivanchik, P. Petitjean, R. Srianand, C. Ledoux, HD molecular lines in an absorption system at redshift $z=2.3377$. Astron. Lett. 27, 683-685 (2001)

J.K. Webb, M.T. Murphy, V.V. Flambaum, V.A. Dzuba, J.D. Barrow, C.W. Churchill, J.X. Prochaska, A.M. Wolfe, Further evidence for cosmological evolution of the fine structure constant. Phys. Rev. Lett. 87, 091301 (2001)

J.K. Webb, J.A. King, M.T. Murphy, V.V. Flambaum, R.F. Carswell, M.B. Bainbridge, Indications of a spatial variation of the fine structure constant. Phys. Rev. Lett. 107, 191101 (2011)

M. Wendt, P. Molaro, QSO 0347-383 and the invariance of $\mathrm{m}_{p} / \mathrm{m}_{e}$ in the course of cosmic time. Astron. Astrophys. 541, 69 (2012)

J.B. Whitmore, M.T. Murphy, Impact of instrumental systematic errors on fine-structure constant measurements with quasar spectra. Mon. Not. R. Astron. Soc. 447, 446-462 (2015)

J.B. Whitmore, M.T. Murphy, K. Griest, Wavelength calibration of the VLT-UVES spectrograph. Astrophys. J. 723, 89-99 (2010)

T. Wilken, G. Lo Curto, R.A. Probst, T. Steinmetz, A. Manescau, L. Pasquini, J.I. Gonzalez Hernandez, R. Rebolo, T.W. Hänsch, T. Udem, R. Holzwarth, A spectrograph for exoplanet observations calibrated at the centimetre-per-second level. Nature 485, 611-614 (2012)

S. Xu, M. Jura, D. Koester, B. Klein, B. Zuckerman, Discovery of molecular hydrogen in white dwarf atmospheres. Astrophys. J. Lett. 766, 18 (2013) 